\title{
The Impact of Patients Losing Their Sense of Smell
}

Olfactory disorders are not as rare as you might think: estimates are that $1-5 \%$ of the population experience it at some point in their lives ${ }^{1}$. It can happen as a result of chronic sinusitis, damage caused by cold viruses, or even a head injury. It is sometimes also a precursor of nervous system diseases such as Parkinson's and Alzheimer's. But compared with hearing and sight loss, it receives little research or medical attention. Losing any sensory input should not be underestimated, this is an argument best reasoned by those who have suffered this loss.

A recent qualitative study by Erskine and Philpott ${ }^{2}$ provided an opportunity to better understand the issues people with smell disorders face. Through the analysis of written, personal accounts of anosmia (loss of sense of smell) sent in to the Norfolk Smell \& Taste Clinic by 71 sufferers, the texts revealed several themes, including feelings of isolation, relationship difficulties, impact on physical health and also the difficulty and cost of seeking help. Many people also commented on the negative attitude from doctors about smell loss, and how they found it difficult to get advice and treatment for their condition.

\section{Significant harm}

Smell loss leaves sufferers vulnerable to environmental hazards, such as spoiled food and gas leaks. It also has a negative effect on a range of activities and experiences, potentially causing significant harm. In reality, perhaps this is not surprising given the extra dimension that smell gives to the enjoyment of food, exploration of our environment and bringing back memories. So, our sense of smell is both a life-saving and life-enhancing sense. Losing it can have the opposite effect. Indeed, there is now accumulating evidence that recent studies from the US and Scandinavia show that anosmia is an independent risk factor for advanced mortality, even when other neurocognitive and cardiovascular factors are controlled for ${ }^{3}{ }^{4}$. Perhaps, given the relative exposure of the olfactory apparatus to the external world through the "unprotected" olfactory neuroepithelium, smell loss acts as a marker for overall neural toxicity. It may therefore be that in future, screening through smell may facilitate health and lifestyle guidance and prognosis.

\section{What it's like to live without a sense of smell}

The aforementioned qualitative study confirmed previous survey findings from $2014^{5}$ that showed that anosmia led to physical concerns included diet and appetite. Because of the reduced pleasure of eating, some participants reported a reduced appetite with subsequent weight loss. Others reported a general decline in the quality of their diet with the reduced perception of flavours leading to an increased intake of foods with low nutritional value (particularly those high in fat, salt and sugar). Many anosmia sufferers complain of a loss of taste as part of their disorder, when in reality they can taste without impairment but have loss the process of retronasal olfaction that occurs during eating. Salt, sweet, sour, bitter and umami are the sensations detected via the taste buds on the tongue and other parts of the mouth and throat; but smell accounts for our $80 \%$ of all flavour perception and as ordinarily, the two sensations coincide, most sufferers assume both sensory modalities have been affected. True gustatory loss accounts for about $1 \%$ of the total workload of a Smell \& Taste clinic, such as the one established at the James Paget University Hospital in Gorleston, Norfolk ${ }^{6}$. 


\section{Emotional disruption}

Emotional negatives experienced by sufferers include embarrassment, sadness, depression, worry and bereavement. The study by Erskine and Philpott showed evidence that it disrupted every aspect of life. These ranged from everyday concerns, such as personal hygiene, to loss of intimacy and the breakdown of personal relationships. Some participants reported that they couldn't take pleasure in occasions that would usually be a cause for celebration. The inability to link smells with happy memories may render these events underwhelming experiences.

Underlying these emotions was the loss of enjoyment of activities, difficulty in expressing the impact of symptoms of anosmia and little sympathy or understanding from outsiders. Others included reduced socialising, no effective treatments and little hope of recovery. Many participants described a profound effect on their relationships with other people as a result of their smell disorder. These range from not enjoying eating together to more intimate relationships - particularly sex.

The financial burdens described included the cost of private referral and alternative treatments. The effects were profound for some, especially if their profession or safety depends upon it. Participants often described negative or unhelpful interactions with GPs and specialists, such as ear, nose and throat surgeons. Participants were concerned by a lack of empathy. Unlike spectacles or hearing aids, no simple solutions are yet available for smell loss. The subject of smell loss remains an enigma to many medical students and doctors, with little training provided on the subject to facilitate appropriate knowledge. Coupled with a relative paucity of known effective treatments for smell loss, this accounts for the relative apathy of the medical profession in comparison to other sensory losses. Study participants and clinic attendees frequently report being told by clinicians that they should consider that their position could be far worse because they could have lost another sense instead!

\section{Support is at hand}

This article will not explore management and treatment of olfactory disorders - this can be found elsewhere ${ }^{7-9}$. However, a key advance in the last decade has been the initiation of a charity to support sufferers of smell and taste disorders. Fifth Sense was established in 2012 by Duncan Boak, an anosmia sufferer and has grown to the point where in 2018 the charity received formal registration from the Charities Commission and most recently secured Lottery Funding support. This latest opportunity will enable Fifth Sense to begin to establish nationwide support hubs in the UK, giving those afflicted with a group of fellow sufferers to share ideas and information, but also partnering with locally engaged clinicians who will have a genuine desire to assess and diagnose the cause of smell loss and advise on any suitable treatment. International guidance is now available through the Position Paper on Olfactory Dysfunction ${ }^{10}$ and the partnership between patients and clinicians will help to move the cause of smell loss sufferers to a more respected place. Fifth Sense website can be found here: www.fifthsense.org.uk 


\section{References}

1. Landis BN, Konnerth CG, Hummel T. A study on the frequency of olfactory dysfunction. Laryngoscope 2004;114(10):1764-9. doi: 10.1097/00005537-200410000-00017

2. Erskine SE, Philpott CM. An unmet need: Patients with smell and taste disorders. Clin Otolaryngol 2020;45(2):197-203. doi: 10.1111/coa.13484 [published Online First: 2019/12/19]

3. Laudisio A, Navarini L, Margiotta DPE, et al. The Association of Olfactory Dysfunction, Frailty, and Mortality Is Mediated by Inflammation: Results from the InCHIANTI Study. J Immunol Res 2019;2019:3128231. doi: 10.1155/2019/3128231 [published Online First: 2019/02/20]

4. Ekstrom I, Sjolund S, Nordin S, et al. Smell Loss Predicts Mortality Risk Regardless of Dementia Conversion. J Am Geriatr Soc 2017 doi: 10.1111/jgs.14770

5. Philpott CM, Boak D. The impact of olfactory disorders in the United kingdom. Chem Senses 2014;39(8):711-8. doi: 10.1093/chemse/bju043

6. Philpott CM. Smell and Taste Disorders in the UK: First experiences with a specialised smell and taste outpatient clinic. Bulletin of the Royal College of Surgeons of England 2014;96:156-59.

7. Philpott C, Addison A. A systematic review of therapeutic options for non-conductive olfactory dysfunction. The Otorhinolaryngologist 2018;11(2):61-71.

8. Syed I, Philpott C. Assessing the sense of smell. British Journal of Hospital Medicine 2015;76(3):C38-C39.

9. Syed I, Philpott C. Hyposmia. Br J Hosp Med (Lond) 2015;76(3):C41-2, C44-5. doi: 10.12968/hmed.2015.76.3.C41

10. Hummel T, Whitcroft KL, Andrews $P$, et al. Position paper on olfactory dysfunction. Rhinology Supplement 2017;54:1-30. 